\title{
APPLICATIONS OF NEW FUZZY INFERENCE-BASED TRACKING LOOPS FOR KINEMATIC GPS RECEIVER*
}

\author{
Wei-Lung Mao ${ }^{1}$
}

\begin{abstract}
Carrier phase measurement is essential for high-accuracy measurement in kinematic global positioning system (GPS) applications. For GPS receiver design, a narrow noise bandwidth is desired to decrease phase jitter due to thermal noise. However, this bandwidth will deteriorate the capability of the tracking loop and result in cycle slipping. Based on bandwidth adjustment criteria, a novel intelligent GPS receiver is proposed for solving the carrier phase tracking problem in the presence of high dynamic environments. A phase error estimator is developed in the carrier loop to conduct the phase error signals; i.e., frequency and frequency ramp errors. Two kinds of fuzzy inference (FI)-based approaches, fuzzy logic control and adaptive neuro-fuzzy control methods, that are simple and have easy realization properties are designed to perform rapid and accurate control of the digital frequency phase-locked loop (FPLL). A new design procedure for kinematic GPS receiver development is also presented. The computer results show that the FI-based receivers achieve faster settling time and wider pull-in range than the conventional tracking loops while also preventing the occurrence of cycle slips.
\end{abstract}

Key words: Global positioning system (GPS), fuzzy logic control, adaptive neuro-fuzzy control, carrier phase tracking, phase-locked loop, frequency-locked loop.

\section{Introduction}

The global positioning system (GPS) has become a commonly-used civil instrument for aircraft precision approaches and in-car navigation systems, etc. Making use of carrier phase information achieves high precision estimation limited to a millimeter level of accuracy. Robust carrier phase tracking has proven to be

* Received November 18, 2005; revised June 20, 2006; Supported by the National Science Council of the Republic of China, Taiwan, under contract no. NSC 94-2213-E-131-004.

1 Department of Electronic Engineering, National Formosa University, No. 64, Wunhua Rd., Huwei Tonwship, Yunlin County, Taiwan 63210, Republic of China. E-mail: wlmao@nfu.edu.tw; wlmao123@yahoo.com.tw 
a challenging task in receiver design, when the trajectories of the users are of high dynamics. In practice, a digital phase-locked loop (DPLL) scheme is always employed in the receiver to extract the phase of the sinusoidal signal. For highly dynamic environments, e.g., in mobile vehicles, the carrier phase signals cannot be followed promptly with conventional tracking loops and result in lost cycles. Once cycle slips occur, several measurement epochs are missed, and a timeconsuming ambiguity search must be restarted. To overcome this notable drawback, some new DPLL designs are expected to improve the positioning accuracy and prevent the occurrence of cycle slipping.

Few researchers have investigated the DPLL design in various kinematic environments. Simon and El-Sherief [5] proposed a fuzzy PLL estimation filter for missile navigation. The gradient descent (GD) algorithm and genetic algorithm (GA) were used for optimization of the fuzzy estimators. However, this target tracking model applies a simplified PLL architecture, which is not an adequate solution of implementation for practical receiver design. Jwo [5] presented an optimal estimator for bandwidth determination in dynamic environments based on a linear Kalman filter. The optimal noise bandwidths were obtained for the first-, second-, and third-order linear PLLs in the steady state. However, if the receivers are utilized in high kinematic circumstances, the linear model assumption no longer holds. Furthermore, the PLL may lose tracking and not return to synchronization operation anymore in the steady state [1]. To date, few studies have attempted to design an intelligent realized receiver when the motion directions exhibit high dynamics. It is a major problem for GPS utilized in a variety of consumer and aerospace applications.

In this paper, a new architecture design for a GPS receiver is proposed to handle robust carrier phase tracking in dynamic environments. The received phase signal comprises five components: (1) phase offset, (2) frequency offset, (3) frequency ramp offset, (4) sinusoidal phase jitter, and (5) decaying phase jitter. To produce an accurate estimate of the phase information, a narrow noise bandwidth must be used. It, in turn, is subjected to a serious problem called cycle slip. A new intelligent carrier loop is presented to deal with this contradiction. The tracking error signals, including (1) phase error, (2) frequency error, and (3) frequency ramp error, are periodically computed and recognized from the phase error estimator. When the phase errors are larger, fuzzy inference (FI) controllers are used to regulate the PLL and frequency-locked loop (FLL) swiftly. Once the tracking errors have decreased, the tracking loop will resolve to the narrowband mode. Because of the simple and flexible structure in digital signal processing (DSP) implementation, fuzzy logic (FL) and adaptive neuro-fuzzy (ANF) methods are presented for intelligent FI controller design. A systematic procedure is also established for robust carrier loop development under narrow bandwidth conditions. Our proposed GPS FI carrier tracking loops are shown to achieve a fast pull-in process and wide locking range in dynamic environments.

The remainder of this paper is organized as follows. In Section 2, the kinematic 
carrier phase model and cycle slip problem are described. Section 3 proposes the FI receiver approach for dynamic environments. A step-by-step digital tracking loop design procedure is presented in Section 4. In Section 5, simulation results are demonstrated to verify the proposed methods. Section 6 concludes this paper.

\section{Kinematic carrier phase model}

The satellite broadcast ranging codes and navigation data at two frequencies: primary L1 (1575.42 MHz) and secondary L2 (1227.42 MHz), and only the L1 signal, free for civilian use, will be considered. The received sample observation is modeled as

$$
r\left(i T_{s}\right)=\sqrt{2 P} D\left[i T_{s}-\tau\right] C A\left[i T_{s}-\tau\right] \cos \left[\omega_{b} i T_{s}+\theta\left(i T_{s}\right)\right]+n_{i},
$$

where $P$ is the average power of the received signal, $D[\cdot]$ is the binary data sequence, $C A[\cdot]$ is the Gold code sequence, $T_{s}=1 / f_{s}$ is the sample period, $\tau$ is the code transmission time delay with respect to the GPS system time, $\omega_{b}$ is the baseband carrier radian frequency, $\theta\left(i T_{S}\right)$ is the unknown carrier phase to be estimated, and $n_{i}$ is the received noise modeled as additive white Gaussian noise (AWGN) with variance $\sigma_{n}^{2}$.

Figure 1 demonstrates the GPS carrier tracking loop. The digitized intermediate frequency (IF) signals are ready to be processed by using a combination of dedicated baseband correlators and a digital signal processor. In order to decode the information, an acquisition method must be used first to detect the presence of the signals. The receiver simultaneously sweeps the uncertainty ranges of the code phase and carrier Doppler frequency shift, so that it can match the incoming and local signals. After the satellite vehicle (SV) code and frequency are successfully replicated during the search process, the carrier and code tracking loops are utilized to synchronize the two-dimensional phases [carrier and pseudo random noise (PRN) code] in the steady state.

The received sequence $r\left(i T_{s}\right)$ is multiplied with the local prompt coarse/acquisition (C/A) code and in-phase/quadrature (I/Q) phase channel correlators. Then the outputs of the in-phase and quadrature-phase accumulators become

$$
\begin{aligned}
I(k T) & =\frac{A}{\sqrt{2}} \cos [\theta(k T)-\hat{\theta}(k T)]+n_{I, k} \\
Q(k T) & =\frac{A}{\sqrt{2}} \sin [\theta(k T)-\hat{\theta}(k T)]+n_{Q, k},
\end{aligned}
$$

where $A=2 \sqrt{P} M D(k T) \sin c\left(\frac{\Delta \omega T}{2}\right) R(\tau-\hat{\tau})$ is the integrated amplitude of the I-phase and Q-phase components [4], $T$ is called the predetection integration time (PIT) or the loop update time, $\Delta \omega=\left(\theta_{k}-\theta_{k-1}\right) / T$, and $M=T / T_{s}$ is the number of samples summed together to update the loop. $\theta(k T)$ and $\hat{\theta}(k T)$ are the carrier phase of the received signal and local carrier signal. $n_{I, k}$ and $n_{Q, k}$ 


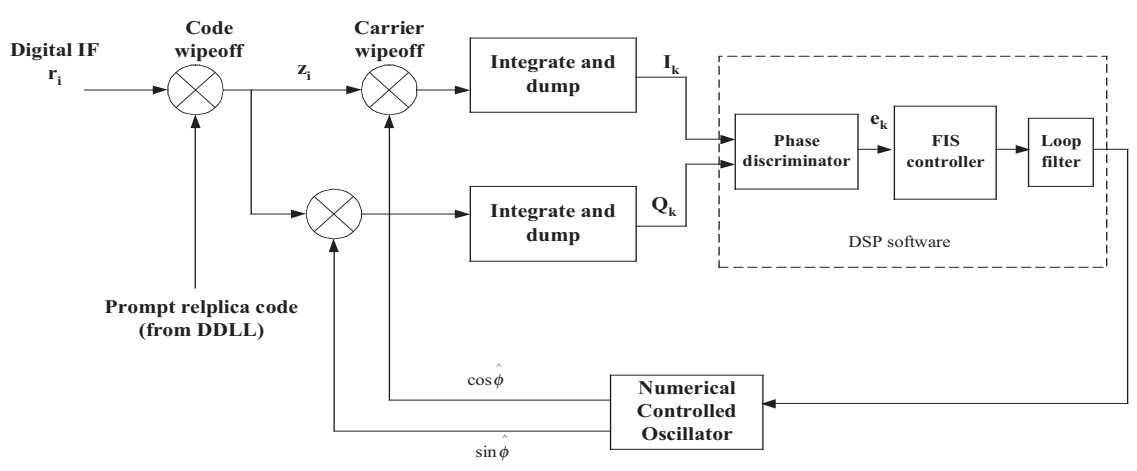

Figure 1. Proposed GPS carrier tracking loop.

are the noise components due to input noise $n_{i}$. The phase error can be computed according to an arc-tangent operation discriminator and we have

$$
\theta_{e}(k T)=\tan ^{-1}\left[\frac{Q(k T)}{I(k T)}\right]=f[\theta(k T)-\hat{\theta}(k T)]+n_{\theta, k},
$$

where $\theta_{e}(k T) \in[-\pi,+\pi]$ is the carrier phase error with the input noise disturbance, and $n_{\theta, k}$ is the phase disturbance noise. If the received sample signal is noise free, $\theta_{e}(k T)=\theta(k T)-\hat{\theta}(k T)$ becomes the phase tracking error in the modulo $[-\pi,+\pi]$ sense.

The satellite and vehicle motion has an impact effect on the processing of the phase and frequency tracking at the GPS receivers. The carrier phase $\theta(k T)$ generally includes five components: (a) phase offset, (b) frequency offset, (c) rate of change of the frequency (frequency ramp) offset, (d) sinusoidal phase jitter, and (e) decaying sinusoidal jitter. The mathematical model for the carrier phase at the sampling instants is

$$
\begin{aligned}
\theta(k T)= & \theta_{0}+\omega_{0}(k T)+\frac{1}{2} \alpha_{0}(k T)^{2}+\sum_{j=0}^{J} A_{j} \sin \omega_{j}(k T) \\
& +\sum_{m=0}^{M} B_{m} e^{-\alpha_{m} k T} \sin \beta_{m}(k T),
\end{aligned}
$$

where $\theta_{0}(\mathrm{rad})$ is simply a fluctuation phase offset difference between the satellite and receiver. $\omega_{0}(\mathrm{rad} / \mathrm{s})$ is the amount of fluctuation radian frequency offset due to the Doppler effect and clock biases. $\alpha_{0}\left(\mathrm{rad} / \mathrm{s}^{2}\right)$ is the amount of Doppler effect and highly dynamic environment between the satellite and mobile vehicle. The phase jitter is typically modeled as sinusoidal waveforms with amplitude $A_{j}$ and frequency $\omega_{j}$. Decaying sinusoidal signals arise in the response of vibration behavior of the kinematic vehicles on the fly. $B_{m}, \alpha_{m}$, and $\beta_{m}$ are the amplitude, decaying factor, and frequency, respectively. The $z$ transform of the input carrier 
phase signal is given by

$$
\begin{aligned}
\theta(z)= & \frac{\theta_{0} z}{z-1}+\frac{2 \pi f_{0} T z}{(z-1)^{2}}+\frac{\pi \alpha_{0} T^{2} z(z+1)}{(z-1)^{3}}+\sum_{j=0}^{J} \frac{A_{j} \sin \omega_{j} T z}{z^{2}-2 \cos \omega_{j} T z+1} \\
& +\sum_{m=0}^{M} \frac{B_{m} e^{-\alpha T} \sin \beta_{m} T z}{z^{2}-2 e^{-\alpha T} \cos \beta_{m} T z+e^{-2 \alpha T}} .
\end{aligned}
$$

For this great diversity of phase components, an intelligent receiver design is required to perform the desired transient and steady response in the carrier tracking loop.

\subsection{Cycle slips}

Due to high phase dynamics and/or phase noise, the carrier transient error will change rapidly. If the phase discriminator cannot recognize the difference between $\theta_{e}(k T)$ and $\theta_{e}(k T)+2 n \pi$ (where $n$ is an integer), then synchronization failure may occur. This phenomenon is called cycle slipping, i.e., jumps of the carrier phase by an integer multiple of the wavelength. If cycle slips occur, they will degrade the high accuracy of the carrier phase range and also that of the position message obtained from such carrier phase observables. Therefore, restarting the carrier phase ambiguity solution is a time- and computation-intensive task and should be avoided as long as possible.

It is desired to maintain lock on the carrier phase to achieve highly accurate kinematic positions. Two limitations of the input frequency are defined as design specifications for kinematic GPS receivers utilized on different vehicles.

(a) Instantaneous frequency step tracking: When the input frequency changes instantaneously, the DPLL may lose lock before the limits of the hold-in range. This is a dynamic limit of the lock operation of a PLL. The frequency step range of the loop is defined as

$$
\omega_{\text {desired }}=\left|\frac{\theta(k T)-\theta(k T-T)}{T}\right| \quad(\mathrm{rad} / \mathrm{s}),
$$

where $\theta(k T)$ and $\theta(k T-T)$ are the carrier phase at $k T$ and $(k-1) T$ instants, respectively. To determine the frequency error unambiguously, the phase difference of two successive values must be less than $\pm \pi$. In this work, the loop update rate is set to $1 \mathrm{kHz}$, and loss of lock is declared when the corresponding instantaneous frequency $\omega_{\text {desired }}$ exceeds $3141.6 \mathrm{rad} / \mathrm{s}$.

(b) Frequent ramp tracking: When the frequency derivative is a constant, the frequency is said to be a ramp signal. It is a difficult problem to maintain lock on the carrier phase, when user acceleration is involved in the tracking loop. The frequency ramp caused by the satellite motion is usually small in GPS, so we only consider the motion of the vehicle [3]. We assume that the user has an acceleration 
of $a_{r}\left(\mathrm{~m} / \mathrm{s}^{2}\right)$, and the corresponding rate of change of the Doppler frequency can be expressed as

$$
\alpha_{\text {desired }}=\left(\frac{a_{r}}{c}\right) \omega_{c} \quad\left(\mathrm{rad} / \mathrm{s}^{2}\right),
$$

where $c$ is the speed of light $\left(=3 \times 10^{8} \mathrm{~m} / \mathrm{s}\right)$ and $\omega_{c}(\mathrm{rad} / \mathrm{s})$ is the GPS L1 channel carrier radian frequency $(=2 \pi \times 1575.42 \mathrm{MHz})$. For example, in a highspeed airplane with an acceleration of $7 \mathrm{G}$ (gravitational acceleration with a value of $9.8 \mathrm{~m} / \mathrm{s}^{2}$ ), the corresponding maximum Doppler frequency ramp $\alpha_{\text {desired }}$ is $2262 \mathrm{rad} / \mathrm{s}^{2}$. This means that the proposed FI receiver must have the capability of tracking the desired instantaneous frequency and frequency ramp without the occurrence of cycle slips.

The thermal noise is another source for skipping a cycle in DPLL. The onesigma thermal noise jitter of a PLL can be calculated as follows [2]:

$$
\sigma_{R}=\frac{360}{2 \pi} \sqrt{\frac{B_{n}}{C N R}\left(1+\frac{1}{2 T * C N R}\right)},
$$

where $\sigma_{R}$ (deg) is the standard deviation of thermal noise, $B_{n}(\mathrm{~Hz})$ is the carrier loop noise bandwidth, and $C N R(\mathrm{~dB}-\mathrm{Hz})$ is the carrier-to-noise ratio for a GPS receiver, typically ranging from $35-55 \mathrm{~dB}-\mathrm{Hz}$ [6]. For a nominal range of CNRs, the thermal noise is always considered insignificant compared to the transient phase error described above. We assume $B_{n}=20 \mathrm{~Hz}$ and $T=0.001 \mathrm{~s}$, and the nominal range of three-sigma thermal noise ranges from $0.024-0.26$ radians. It is observed that cycle slipping due to a high dynamic trajectory can be the dominant factor in kinematic environments.

\subsection{Noise bandwidth}

In carrier loop design, a trade-off between two contradicting considerations is always an issue. This involves the selection of the noise bandwidth. The thermal noise jitter is sensitive to the noise bandwidth. Decreasing the noise bandwidth can reduce the thermal noise. Hence, it will, in turn, reduce the ability of the loop to track the Doppler-shifted carrier phase signal induced by the user dynamics. In practice, the carrier noise bandwidths are designed in the range of 5-20 Hz for a GPS receiver to suppress the thermal noise jitter. The pull-out frequency and frequency ramp ranges [1] of a conventional PLL can be obtained as

$$
\begin{aligned}
& \omega_{P O}=1.8 \omega_{n}(\xi+1) \\
& \alpha_{P O}=\omega_{n}^{2},
\end{aligned}
$$

where $\omega_{n}(\mathrm{rad} / \mathrm{s})$ is the natural frequency, and $\xi$ is the damping ratio. The loop can maintain lock for a frequency step $\omega<\omega_{P O}$ and frequency ramp $\alpha<\alpha_{P O}$. For the nominal range of noise bandwidth, the pull-out ranges are usually narrow. It is difficult to maintain lock for a narrow bandwidth PLL that suffers from 
severe Doppler environments. The next section proposes a new tracking loop to overcome this paradox.

\section{Fuzzy inference-based (FIB) receiver}

The baseband structure of the proposed intelligent receiver model applied in dynamic environments is shown in Figure 2a. The carrier loop is composed of a phase detector with modulo function, a phase error estimator, FI-based controllers, digital filters, and a numerically controlled oscillator (NCO). Because of the simple architecture in the realization aspect and the nonlinear property of the closed-loop system, two FI schemes, namely fuzzy logic control and adaptive neuro-fuzzy control, are utilized to design the intelligent controllers. Two controllers, FI controller 1 and FI controller 2, are employed to control the secondorder PLL and assisted second-order FLL, respectively.

\subsection{Phase error estimator}

The control flowchart of the proposed receiver is depicted in Figure $2 \mathrm{~b}$. When the code and carrier loop enters the tracking mode, the frequency error $\omega_{e, k}$ and frequency ramp error $\alpha_{e, k}$ are periodically computed by the phase error estimator and are given by

$$
\begin{aligned}
& \omega_{e, k}=\theta_{e, k}-\theta_{e, k-1} \\
& \alpha_{e, k}=\omega_{e, k}-\omega_{e, k-1} .
\end{aligned}
$$

\subsection{Fuzzy logic controller (FLC)}

In our system, the state vector $\mathbf{X}_{1, k}=\left[\begin{array}{ll}\theta_{e, k} & \omega_{e, k}\end{array}\right]^{T}$ and $\mathbf{X}_{2, k}=\left[\begin{array}{ll}\theta_{e, k} & \alpha_{e, k}\end{array}\right]^{T}$ are chosen as the input variables of FI controller 1 and FI controller 2, shown in Figure 3. Five fuzzy terms are defined for each linguistic variable. These terms are negative big (NB), negative small (NS), zero (ZE), positive small (PS), and positive big (PB). We choose triangular-shaped membership functions for the control input and output variables, and the input and output membership functions are depicted in Figure 3a. An inference mechanism based on the Mamdani algorithm is evaluated and implemented here. The fuzzy rule tables providing the human knowledge base of the system are depicted in Figure 3b. These rules are expressed as

$$
\begin{aligned}
R^{i}: \text { If } x_{1} \text { is } A_{1}^{i}, \quad \text { and } & x_{2} \text { is } A_{2}^{i}, \\
\text { then } y \text { is } B^{i} . & R^{i} i=1, \ldots, 50,
\end{aligned}
$$




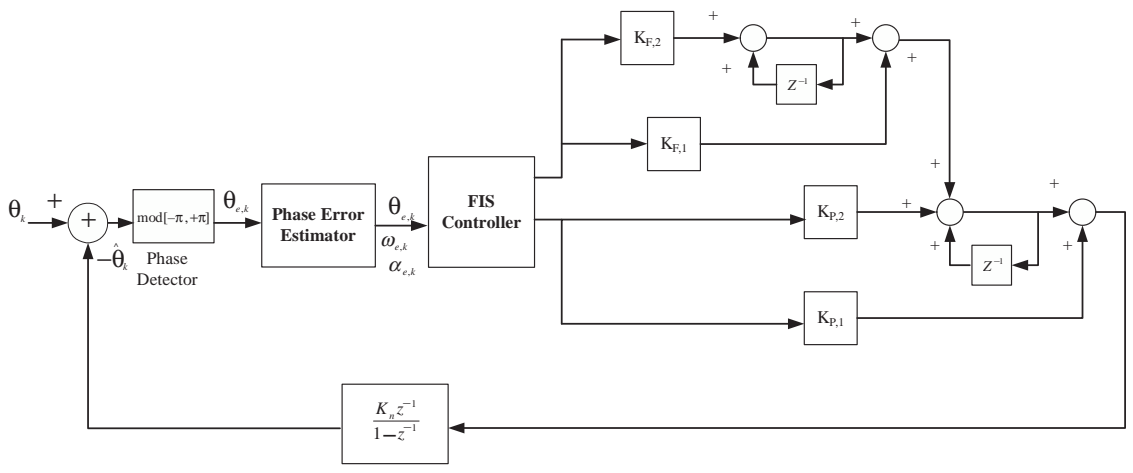

(a)

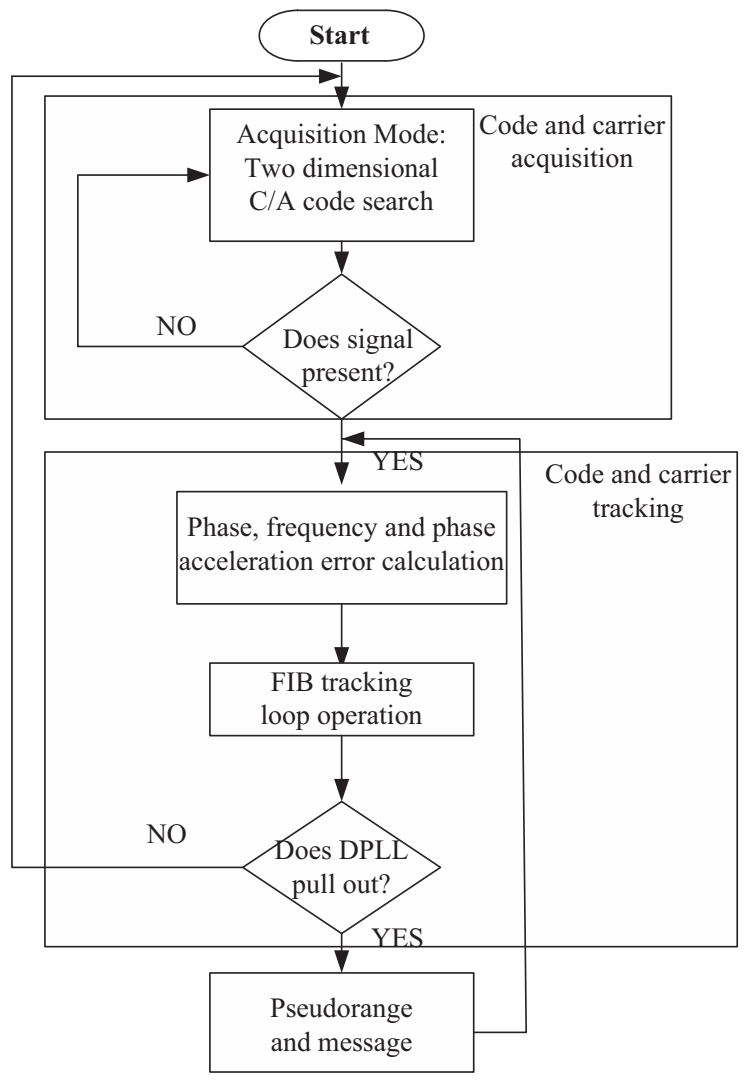

(b)

Figure 2. (a) Proposed baseband model of FI-based FPLL, (b) control flowchart for GPS receiver. 

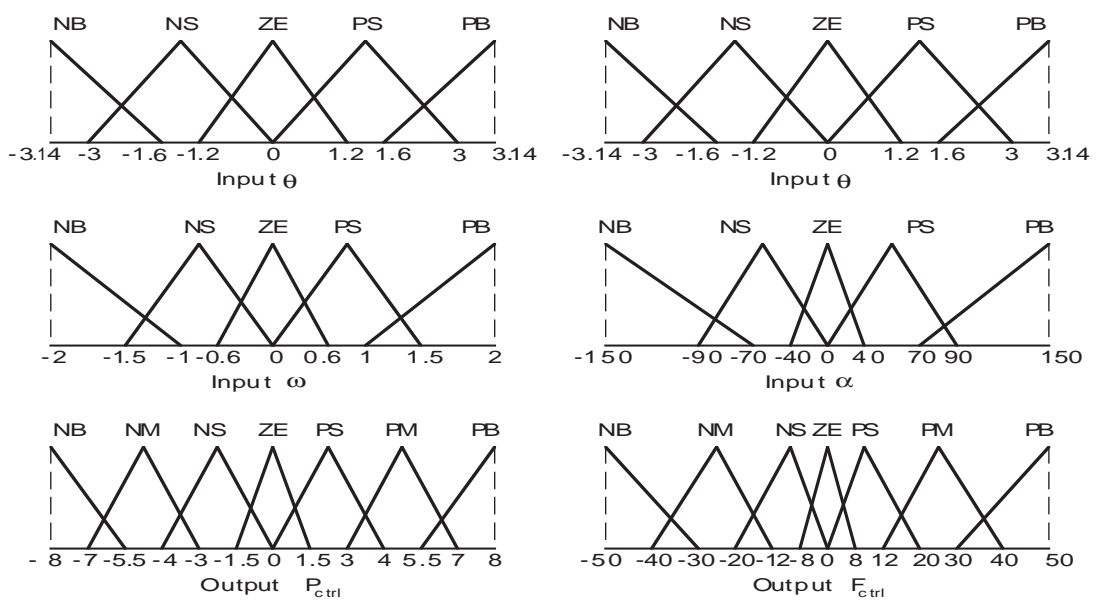

(a)

\begin{tabular}{|c|c|c|c|c|c|}
\hline$\theta^{\omega}$ & NB & NS & ZE & PS & PB \\
\hline NB & NB & NB & NM & NM & NM \\
\hline NS & NB & NM & NM & NS & NS \\
\hline ZE & NM & NS & ZE & PS & PM \\
\hline PS & PS & PS & PM & PM & PB \\
\hline PB & PM & PM & PM & PB & PB \\
\hline
\end{tabular}

\begin{tabular}{|c|c|c|c|c|c|}
\hline$\alpha$ & NB & NS & ZE & PS & PB \\
\hline NB & NB & NB & NB & NM & NM \\
\hline NS & NB & NM & NM & NM & NS \\
\hline ZE & NM & NS & ZE & PS & PM \\
\hline PS & PS & PM & PM & PM & PB \\
\hline PB & PM & PM & PB & PB & PB \\
\hline
\end{tabular}

(b)

Figure 3. (a) Membership functions, (b) fuzzy rule tables for FI controller 1 and FI controller 2, respectively.

where $x_{1}, x_{2}$, and $y$ are linguistic variables, and $A_{1}^{i}, A_{2}^{i}$, and $B^{i}$ are linguistic labels (or fuzzy sets) characterized by membership functions. The fuzzy output is determined from the center average defuzzification formulated as follows:

$$
\bar{y}=\frac{\sum_{i=1}^{n} y_{i} u\left(y_{i}\right)}{\sum_{i=1}^{n} u\left(y_{i}\right)},
$$

where $n$ is the number of fuzzy output sets, $y_{i}$ is the numerical value of the $i$ th output membership function, and $u\left(y_{i}\right)$ represents its membership value at the $i$ th quantization level. 


\subsection{Adaptive neuro-fuzzy controllers (ANFCs)}

The ANF is a five-layer feedforward network (see Figure 4a), where each layer performs a particular node function on incoming signals. It is assumed that the FI system uses a first-order Sugeno model with if-then rules in the following form:

$$
\begin{aligned}
& R^{j}: \text { If } x_{1} \text { is } A_{1}^{j} \text { and } x_{2} \text { is } A_{2}^{j}, \\
& \text { then } f_{j}=a_{0}^{j}+a_{1}^{j} x_{1}+a_{2}^{j} x,
\end{aligned}
$$

where $x_{i}$ is the input linguistic variable, $f_{j}$ is the output consequent variable, $A_{i}^{j}$ are the linguistic labels associated with membership functions $\mu_{A_{i}^{j}}\left(x_{i}\right), a_{i}^{j}$ are called the consequent parameters of linear equations $f_{j}$, and $j=1,2, \ldots, M$. We select the membership to be a Gaussian function, such as

$$
\mu_{A_{i}^{j}}(x)=\exp \left[-\left(\frac{x-m_{i}^{j}}{\sigma_{i}^{j}}\right)^{2}\right],
$$

where $m_{i}^{j}$ and $\sigma_{i}^{j}$ are the mean and standard deviation of the Gaussian membership function, respectively. They are referred to as premise parameters. The functions of the five layers of the ANFC are described as follows.

Layer 1. Each node in this layer corresponds to one linguistic label of an input variable, and its output specifies the degree to which the given $x_{i}$ belong to a fuzzy set $A_{i}^{j}$. The outputs of this node function are

$$
O_{1, i}^{j}=\mu_{A_{i}^{j}}\left(x_{i}\right) \quad i=1,2,3 .
$$

Layer 2. Every node in this layer multiplies the incoming signals and delivers the product out. Each fuzzy rule is performed for a node and produces the firing strength of a rule. The function of each output node uses the following AND operation:

$$
O_{2}^{j}=w_{j}=\mu_{A_{1}^{j}}\left(x_{1}\right) * \mu_{A_{2}^{j}}\left(x_{2}\right) \quad j=1, \ldots, M .
$$

Layer 3. Each node in this layer computes the normalized firing strength of a rule. The $j$ th node calculates the ratio of the $j$ th rule's firing strength to the sum of all the rules' firing strengths:

$$
O_{3}^{j}=\bar{w}_{j}=\frac{w_{j}}{\sum_{j=1}^{M} w_{j}} .
$$

Layer 4. Each node in this layer calculates the weighted consequent value for a rule. The function of each node performs the algebra product:

$$
O_{4}^{j}=\bar{w}_{j} f_{j}=\bar{w}_{j}\left(a_{0}^{j}+a_{1}^{j} x_{1}+a_{2}^{j} x_{2}\right) .
$$




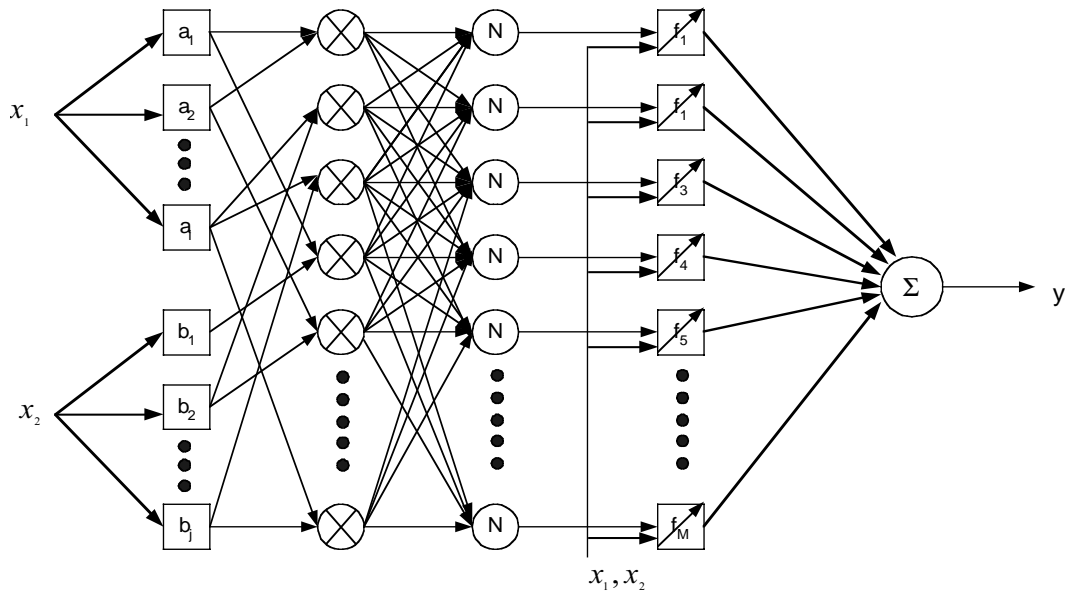

(a)
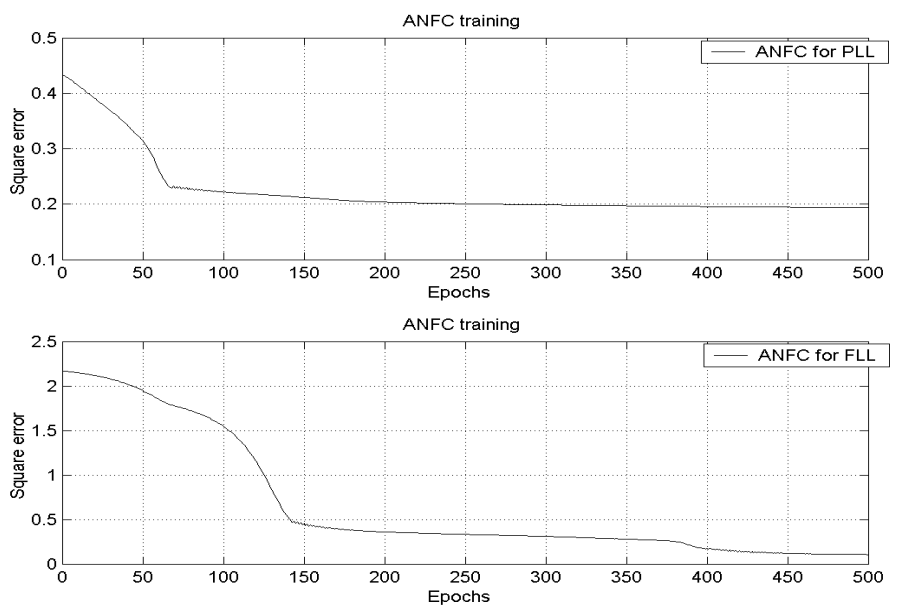

(b)

Figure 4. (a) ANFC model, (b) training error curves with RLS algorithm for FI controllers 1 and 2.

Layer 5. The single node in this layer sums the overall incoming signals to the final inferred result for the ANFC:

$$
O_{5}=y^{*}=\sum_{j=1}^{M} O_{4}^{j}=\sum_{j=1}^{M} \bar{w}_{j} f_{j} .
$$

The ANFC has two sets of adjustable parameters, i.e., premise and consequent parameters. For simplicity, only consequent parameters are adapted here. From 
equation (15.5), the final inferred output is a linear function of the consequent parameter set $\left\{a_{0}^{j}, a_{1}^{j}, a_{2}^{j}\right\}$. Given the membership parameters and the training data pairs, the $p$ linear equations in terms of consequent parameters are given by

$$
\begin{gathered}
{\left[\begin{array}{ccccccc}
\bar{w}_{1}^{(1)} & \bar{w}_{1}^{(1)} x_{1}^{(1)} & \bar{w}_{1}^{(1)} x_{2}^{(1)} & \ldots & \bar{w}_{M}^{(1)} & \bar{w}_{M}^{(1)} x_{1}^{(1)} & \bar{w}_{M}^{(1)} x_{2}^{(1)} \\
\vdots & \vdots & \vdots & \vdots & \vdots & \vdots & \vdots \\
\bar{w}_{1}^{(p)} & \bar{w}_{1}^{(p)} x_{1}^{(p)} & \bar{w}_{1}^{(p)} x_{2}^{(p)} & \cdots & \bar{w}_{M}^{(p)} & \bar{w}_{M}^{(p)} x_{1}^{(p)} & \bar{w}_{M}^{(p)} x_{2}^{(p)}
\end{array}\right]} \\
\times\left[\begin{array}{c}
a_{0}^{1} \\
a_{1}^{1} \\
a_{2}^{1} \\
\vdots \\
a_{0}^{M} \\
a_{1}^{M} \\
a_{2}^{M}
\end{array}\right]=\left[\begin{array}{c}
d^{(1)} \\
d^{(2)} \\
\vdots \\
d^{(p)}
\end{array}\right] .
\end{gathered}
$$

For notational simplicity, equation (16.1) can be described as a matrix form:

$$
\mathbf{C X}=\mathbf{D}
$$

where $\mathbf{C}=\left[\begin{array}{llll}\mathbf{c}_{1}^{T} & \mathbf{c}_{2}^{T} & \cdots & \mathbf{c}_{p}^{T}\end{array}\right]^{T}$ is a $p$ by $(3 * M)$ data matrix, $\mathbf{c}_{n}$ is a 1 by $(3 * M)$ row vector, $\mathbf{D}$ is a $p$ by 1 desired data vector, and $\mathbf{X}$ is a $(3 * M)$ by 1 unknown weight vector represented by arranging all the consequent parameters in one column vector. We adopt the recursive least squares (RLS) algorithm to rapidly train and adapt the consequent parameters of the equivalent FI system. The RLS algorithm can be expressed as

$$
\begin{aligned}
\mathbf{G}_{n+1} & =\frac{\lambda^{-1} \mathbf{P}_{n} \mathbf{c}_{n+1}^{T}}{1+\lambda^{-1} \mathbf{c}_{n+1} \mathbf{P}_{n} \mathbf{c}_{n+1}^{T}} \\
\xi_{n+1} & =d^{(n+1)}-\mathbf{c}_{n+1} \hat{\mathbf{X}}_{n} \\
\hat{\mathbf{X}}_{n+1} & =\hat{\mathbf{X}}_{n}+\mathbf{G}_{n+1} \xi_{n+1} \\
\mathbf{P}_{n+1} & =\lambda^{-1} \mathbf{P}_{n}-\lambda^{-1} \mathbf{K}_{n+1} \mathbf{c}_{n+1} \mathbf{P}_{n} \quad n=0,1, \ldots, p-1,
\end{aligned}
$$

where $\hat{\mathbf{X}}_{n}$ is the estimated consequent weight vector. The matrix $\mathbf{P}_{n}$ is referred to as an inverse correlation matrix, and $\lambda$ is a forgetting factor. The time-varying vector $\mathbf{G}_{n}$ called the gain vector can update the value itself according to equation (17.1). Additionally, we need to specify the initial conditions of the RLS algorithm to start the learning process. The weight vector $\hat{\mathbf{X}}_{0}$ is set as a zero vector, and the inverse correlation matrix $\mathbf{P}_{n}$ is set as $\mathbf{P}_{0}=\delta^{-1} \mathbf{I}$, where the positive constant $\delta$ is the only parameter required for this initialization. 


\section{Digital tracking loop design}

The filter order and noise bandwidth determine the loop response to signal dynamics and become two vital factors in the carrier loop. A second-order PLL is unconditionally stable at all noise bandwidths but suffers from sensitivity to acceleration stress error. In our approach, an assisted second-order FLL is applied to improve the phase acceleration dynamics. Table 1 illustrates the detailed transformation between analog and digital PLLs based on the backward Euler method (BEM). It is obtained by using a numerical method to approximate the integral equation. The closed-loop transfer function of a second-order PLL/FLL can be represented as

$$
H(s)=\frac{a \omega_{n} s+\omega_{n}^{2}}{s^{2}+a \omega_{n} s+\omega_{n}^{2}},
$$

where $\omega_{n}(\mathrm{rad} / \mathrm{s})$ is the natural frequency, and $a$ is the filter coefficient. The singlesided loop noise bandwidth $B_{n}$ for this system is

$$
B_{n}=\frac{1}{|H(0)|^{2}} \int_{0}^{\infty}|H(j 2 \pi f)|^{2} d f=\left(\frac{1+4 a^{2}}{4 a}\right) \omega_{n} \quad(\mathrm{~Hz}) .
$$

The natural frequency $\omega_{n}$ can be determined in terms of the desired loop bandwidth $B_{n}$. Because most GPS carrier tracking loops are built as a digital structure, the BEM is utilized to map the continuous-time system into a discrete-time one. We herein consider the first-order loop filter with a transfer function:

$$
F(z)=K_{1}+K_{2} \frac{1}{1+z^{-1}},
$$

where $K_{1}$ and $K_{2}$ are digital filter coefficients of the DPLL/DFLL. We assume that $K_{d}$ is the gain of the phase discriminator and $K_{n}$ is the gain of the NCO. The equivalent mathematical model of the $\mathrm{NCO}$ is written as

$$
N(z)=\frac{K_{n} z^{-1}}{1-z^{-1}} .
$$

Inserting equations (20) and (21) into the carrier tracking system, the discretetime closed-loop transfer function becomes

$$
H(z)=\frac{K_{d} K_{n} z^{-1}\left(K_{1}+K_{2}-K_{1} z^{-1}\right)}{1+\left(K_{d} K_{n} K_{1}+K_{d} K_{n} K_{, 2}-2\right) z^{-1}+\left(1-K_{d} K_{n} K_{1}\right) z^{-2}}
$$

Starting from an analog equivalent transfer function such as equation (18), we can derive the corresponding digital loop filter coefficients $K_{1}$ and $K_{2}$ as

$$
\begin{aligned}
& K_{1}=\frac{\sqrt{2} \omega_{n} T}{K_{d} K_{n}} \\
& K_{2}=\frac{\omega_{n}^{2} T^{2}}{K_{d} K_{n}} .
\end{aligned}
$$


In our design, a narrower noise bandwidth $(\sim 20 \mathrm{~Hz})$ is adopted to maintain an accurate position and velocity determination. Nonlinear FI controllers acting as variable gains are employed to follow the incoming phase promptly in a highly dynamic environment. The proposed design processes of the kinematic GPS receiver are the following steps.

Step 1. Determine the desired instantaneous frequency step and frequency ramp limitations of a moving vehicle carrying a GPS receiver from equations (6) and (7). The maximum Doppler velocity and acceleration of various mobile users must be obtained first.

Step 2. Choose the carrier noise bandwidth $B_{n}$. In practice, a narrow bandwidth of range $5-20 \mathrm{~Hz}$ is chosen. This is one set of several possible selections that the carrier loop can operate.

Step 3. Determine the gains of the phase discriminator $\left(K_{d}\right)$ and NCO $\left(K_{n}\right)$. The damping ratio is selected as the optimum value 0.707 . The corresponding filter parameters $K_{1}$ and $K_{2}$ are found from equations (23.1) and (23.2).

Step 4. Design the proposed FI controllers for the FPLL. The FLC and ANFC schemes are the two candidates employed to perform the robust carrier phase tracking.

Step 5. Perform the dynamic transient tests for different trajectories at a low CNR of $35 \mathrm{~dB}-\mathrm{Hz}$. Five types of carrier phase signals are considered to conduct each algorithm. Because the carrier tracking loop is nonlinear near the threshold region, a Monte Carlo simulation under the dynamic and CNR conditions will determine the true tracking performance of the GPS receivers.

Step 6. Examine the transient and steady-state performances in a variety of dynamics. By adapting the rule tables and patterns correctly, the FI controllers of the system can be obtained with respect to the desired limitations above. Some other performance indexes, e.g., settling time and locking ranges, can also be considered.

\section{Simulation results}

To demonstrate the characteristics of the proposed FI-based GPS receiver, several computer simulation results in five different circumstances are performed. We consider tracking the L1 carrier frequency $(1575.42 \mathrm{MHz})$ in this work. The received signal is bandpass filtered, amplified, and down-converted to an intermediate frequency (IF) and then digitized. The IF is fixed at $1.25 \mathrm{MHz}$, and a sampling frequency of $5 \mathrm{MHz}$ is selected. Typically, the CNRs for the GPS receiver range from $35-55 \mathrm{~dB}-\mathrm{Hz}$ [2], and can be defined by

$$
C N R=10 \log (S N R * B)=10 \log \left(\frac{P}{\sigma_{n}^{2} * T_{S}}\right) \quad[\mathrm{dB}-\mathrm{Hz}],
$$


Tracking LOOPS FOR KINEMATIC GPS RECEIVER 105

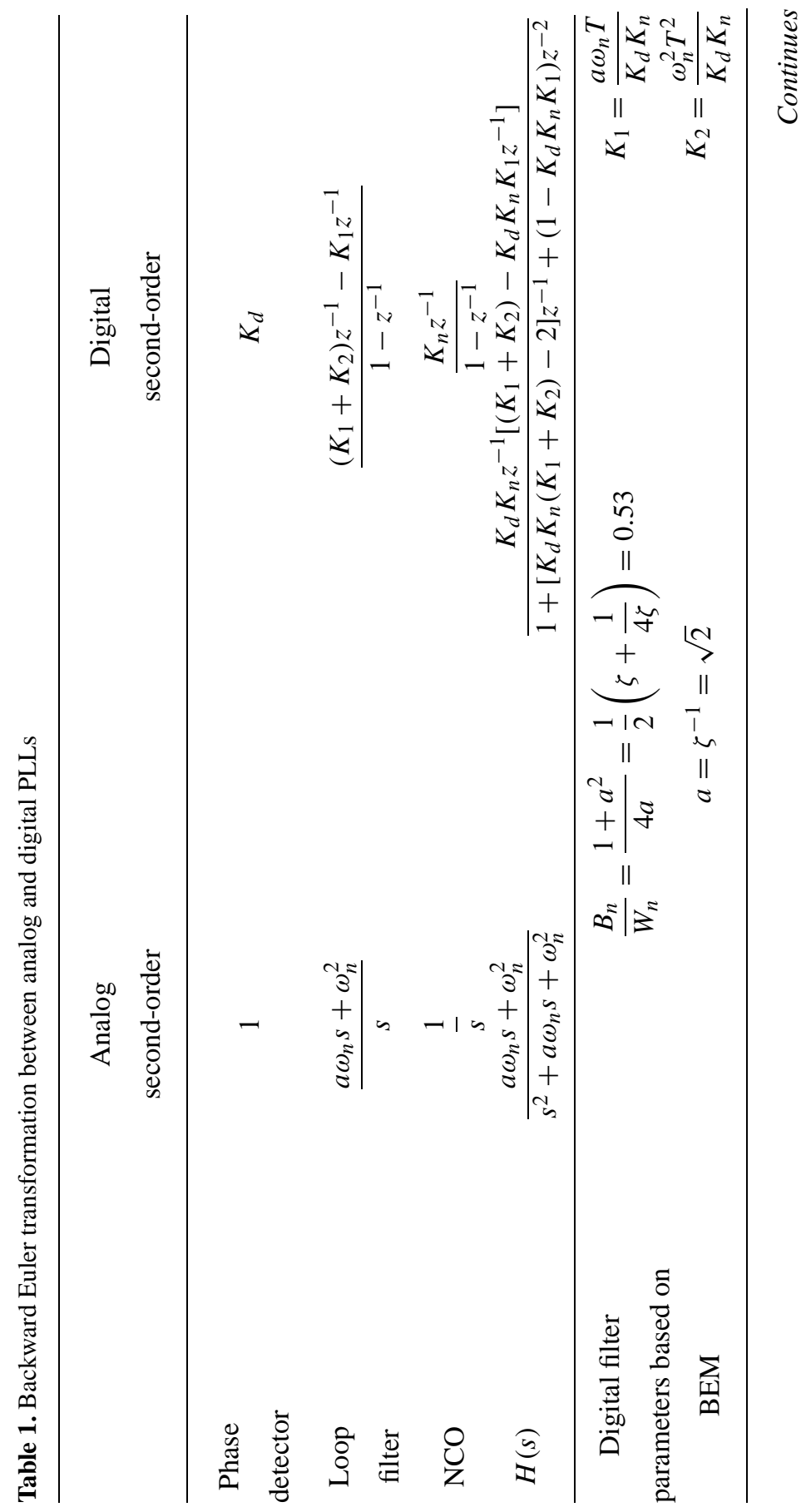


106 MAO

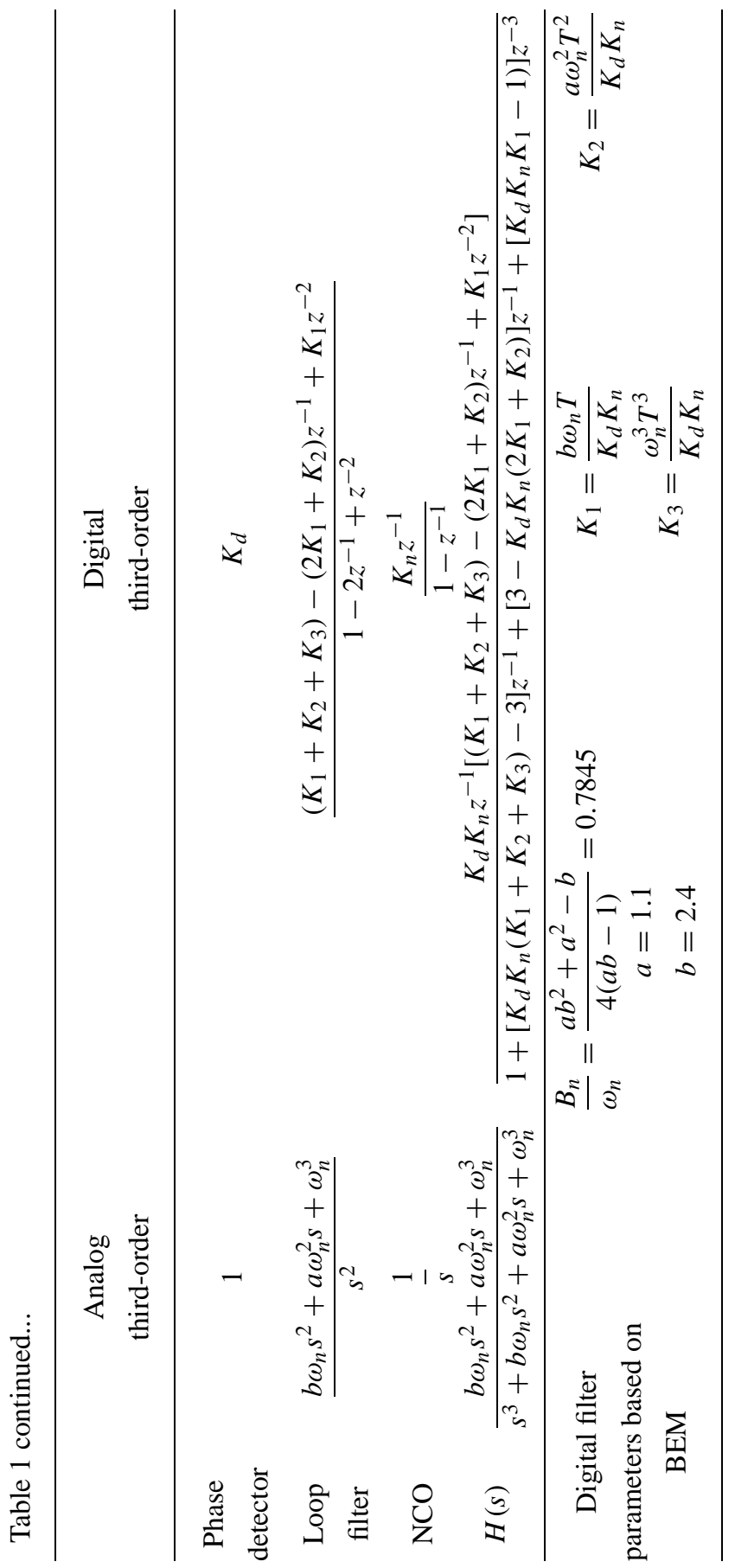


Table 2. Digital filter parameters of second-order and third-order DPLL ( $K_{d}=1, K_{n}=$ $400 \pi$ )

\begin{tabular}{lll}
\hline DPLL order & Noise BW $(\mathrm{Hz})$ & Digital filter parameters \\
\hline Second-order & 20 & $K_{1}=4.247 \mathrm{e}-5, K_{2}=1.133 \mathrm{e}-6$ \\
\hline Third-order & 18 & $K_{1}=4.382 \mathrm{e}-5, K_{2}=4.608 \mathrm{e}-7, K_{3}=9.612 \mathrm{e}-9$
\end{tabular}

(a)

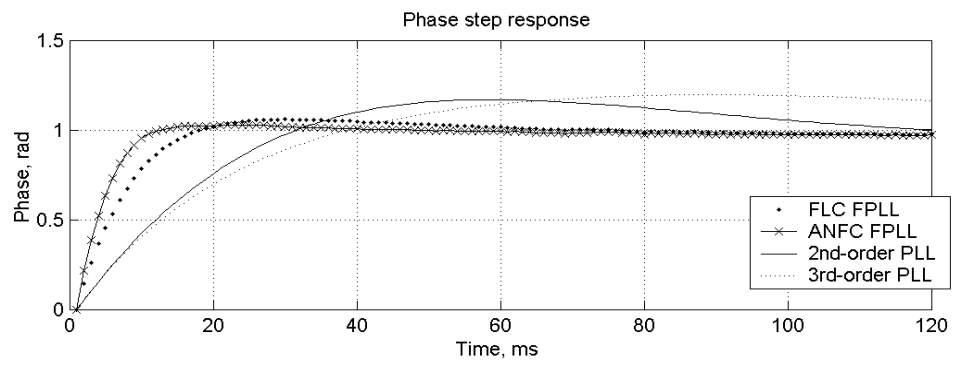

Phase step error

(b)

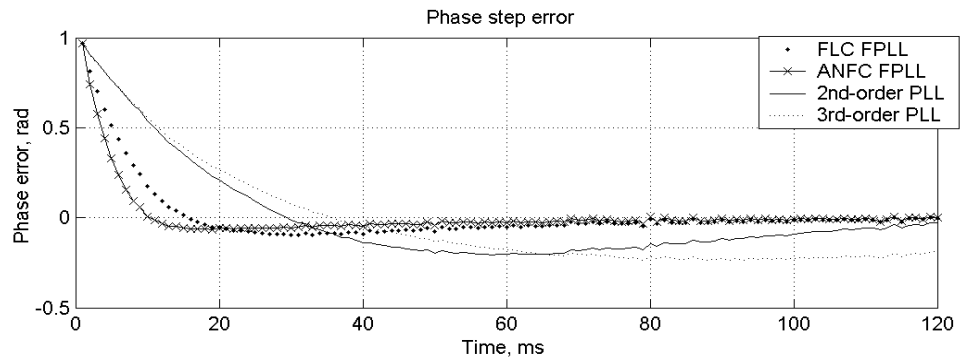

Figure 5. (a) Phase step responses, (b) phase errors for each FLC FPLL, ANFC FPLL, second-order PLL, and third-order PLL when input phase offset is 1 radian.

where $P$ is the power of the received signal assumed to be unity without loss of generality (from equation (1)), $\sigma_{n}^{2}$ is the power of AWGN noise, and $T_{s}=0.2 \mathrm{~ms}$ is the sampling period. The DPLL dynamic tests here are conducted for a low value of $\mathrm{CNR}$ of $35 \mathrm{~dB}-\mathrm{Hz}$, and each curve is obtained based on 1000 independent Monte Carlo simulations.

Four types of DPLLs are compared, namely FLC FPLL, ANFC FPLL, conventional second-order PLL, and third-order PLL. The complete fuzzy rules for FL controllers are illustrated in Figure 3. For five linguistic terms used for each input variable, 25 rules are developed for each controller. Each ANFC also contains 25 rules with five membership functions being assigned to each input variable, and the total number of consequent parameters is 75 . In Step 1 above, three desired dynamic signals: a phase offset of $\pi / 2$ radian, an instantaneous frequency step of 
(a)

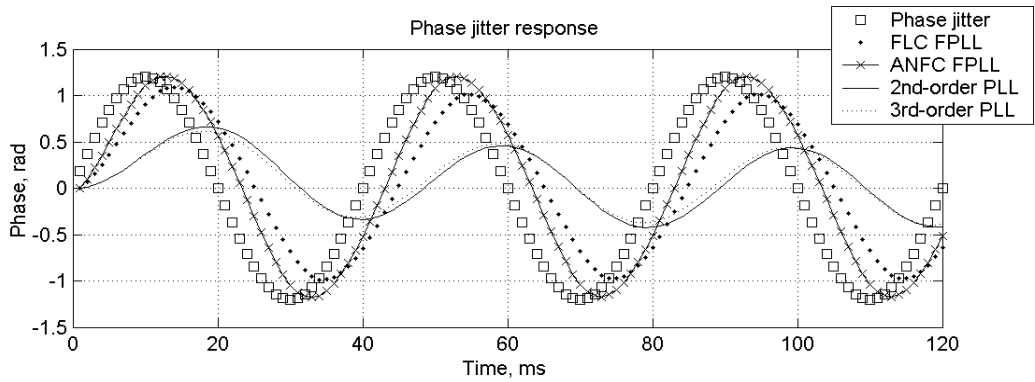

(b)

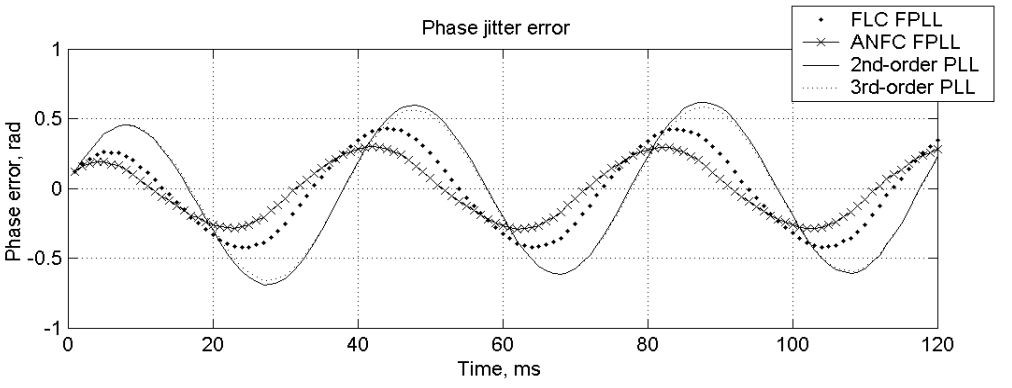

Figure 6. (a) Sinusoidal phase jitter responses, (b) phase errors for each FLC FPLL, ANFC FPLL, second-order PLL, and third-order PLL when jitter frequency is $25 \mathrm{~Hz}$ with $A_{1}=1.2$.

$400 \mathrm{~Hz}$, and a frequency ramp of $400 \mathrm{~Hz} / \mathrm{s}$, are utilized as the reference training waveforms. We collect the effective input-output information from each of three dynamic environments individually developed in the FLC, and the patterns are joined together to apply in the ANF network training procedure. Figure $4 \mathrm{~b}$ shows the training error curves of two ANFCs with the RLS algorithm. The noise bandwidths for traditional second- and third-order loops are chosen as $20 \mathrm{~Hz} / 18 \mathrm{~Hz}$ consistent with stability, and the corresponding digital filter parameters are summarized in Table 2.

Figures $5 \mathrm{a}$ and $\mathrm{b}$ illustrate the phase offset and phase error responses of each scheme, when a 1 radian step input is applied. The total simulation time is 120 $\mathrm{ms}$. It is seen that the ANFC and FLC FPLL achieve a better performance in terms of settling times of $30 \mathrm{~ms}$ and $60 \mathrm{~ms}$ for the 5\% phase error specification, respectively. On the other hand, the conventional second- and third-order PLLs require settling times of $115 \mathrm{~ms}$ and $175 \mathrm{~ms}$, approximately. It appears that the proposed methods do enhance the PLL to track the carrier phase rapidly.

In a dynamic environment, the carrier signal contains phase fluctuations because of the channel fading. The performances of each tracking loop for phase jitter tracking are demonstrated in Figure 6. Here, a single-tone sinusoid corresponding to a jitter frequency of $25 \mathrm{~Hz}$ with $A_{1}=1.2$ is considered. It is observed that FI-based FPLLs can handle the tracking of jitter waveforms promptly on 
(a)

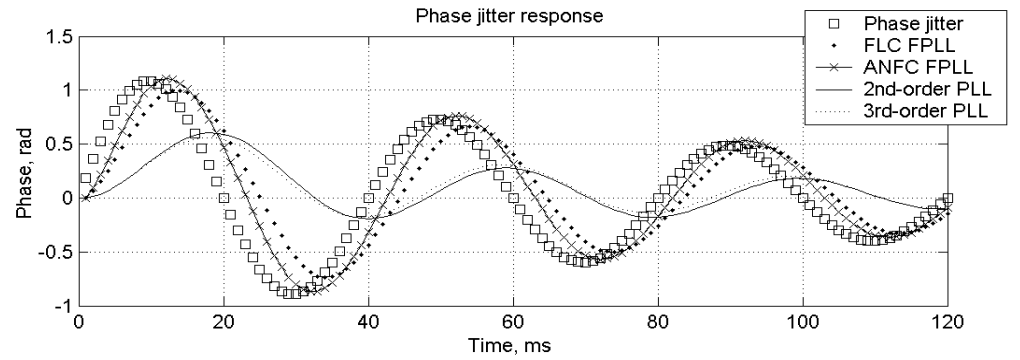

(b)

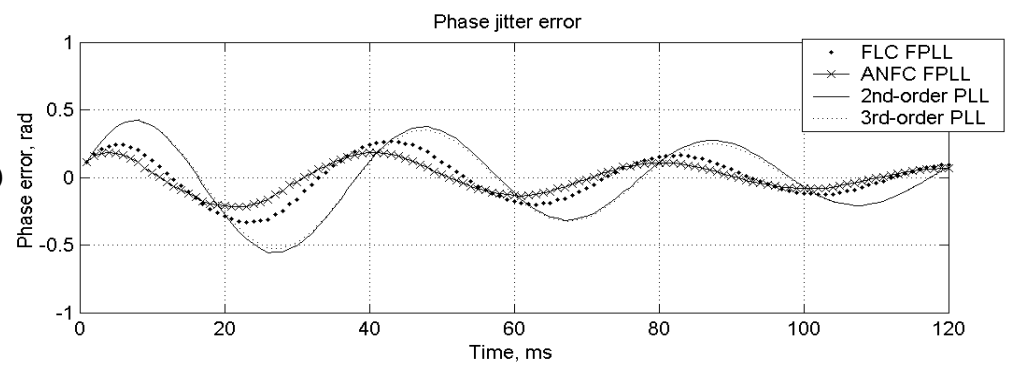

Figure 7. (a) Decaying sinusoidal jitter responses, (b) phase errors for each FLC FPLL, ANFC FPLL, second-order PLL, and third-order PLL when jitter frequency is $25 \mathrm{~Hz}$ with $B_{1}=1.2$ and $\alpha_{1}=0.1$.

both amplitude and phase. In the steady state, the phase error residuals of the conventional PLLs are more than 0.5 radian. Examples of decaying phase jitter on the tracking loops are plotted in Figures $7 \mathrm{a}$ and $\mathrm{b}$. The results are similar to those in the case of the sinusoidal jitter. The transient responses of the proposed schemes outperform those of the traditional PLLs with a faster convergence rate.

Figures $8 \mathrm{a}$ and $\mathrm{b}$ are the system responses when an instantaneous $400 \mathrm{~Hz}$ frequency step is applied. In this dynamic circumstance, both FI controller 1 and controller 2 are utilized to control the dual-loop FPLL. It is shown from Figure 8a that the unlocked phenomenon occurs in the traditional second- and third-order loops. The pull-in process is rather slow, so a long pull-in time is required to keep lock again. The same figure shows that an excellent phase tracking performance has been obtained and the control loops are locked all the time by the FI methods. The FLC and ANFC schemes require settling times of $15 \mathrm{~ms}$ and $12 \mathrm{~ms}$ approximately, achieving the 5\% frequency error specification. Table 3 shows the results of settling time versus Doppler frequency shift for each loop scheme, in which the frequency step $f_{0}$ changes from $50 \mathrm{~Hz}$ to $400 \mathrm{~Hz}$. Our proposed receiver shows a significant improvement in terms of shorter settling time than other conventional loops. As can been seen, the cycle slipping phenomenon occurs in the conventional loops as the frequency offset becomes larger than $100 \mathrm{~Hz}$. By incorporating 
110 MAO

Table 3. Settling times for each digital carrier loop under Doppler frequency environments

\begin{tabular}{|c|c|c|c|c|c|c|c|c|}
\hline $\begin{array}{l}\text { Frequency } \\
\text { step }(\mathrm{Hz})\end{array}$ & 50 & 100 & 150 & 200 & 250 & 300 & 350 & 400 \\
\hline $\begin{array}{c}\text { Types of digital } \\
\text { carrier loop }\end{array}$ & \multicolumn{8}{|c|}{ Settling time (ms) } \\
\hline FLC FPLL & 4 & 4 & 4 & 6 & 8 & 11 & 13 & 15 \\
\hline ANFC FPLL & 4 & 4 & 5 & 5 & 6 & 9 & 11 & 12 \\
\hline Second-order PLL & 119 & $\begin{array}{l}221 \\
(\mathrm{CS})\end{array}$ & $\begin{array}{l}432 \\
(\mathrm{CS})\end{array}$ & $\begin{array}{l}2113 \\
(\mathrm{CS})\end{array}$ & $\mathrm{LL}$ & LL & $\mathrm{LL}$ & LL \\
\hline Third-order PLL & 513 & $\begin{array}{l}630 \\
(\mathrm{CS})\end{array}$ & $\begin{array}{l}1132 \\
(\mathrm{CS})\end{array}$ & $\begin{array}{l}1862 \\
(\mathrm{CS})\end{array}$ & $\begin{array}{l}2288 \\
(\mathrm{CS})\end{array}$ & LL & LL & $\mathrm{LL}$ \\
\hline
\end{tabular}

CS: cycle slips, LL: loss of lock.

(a)

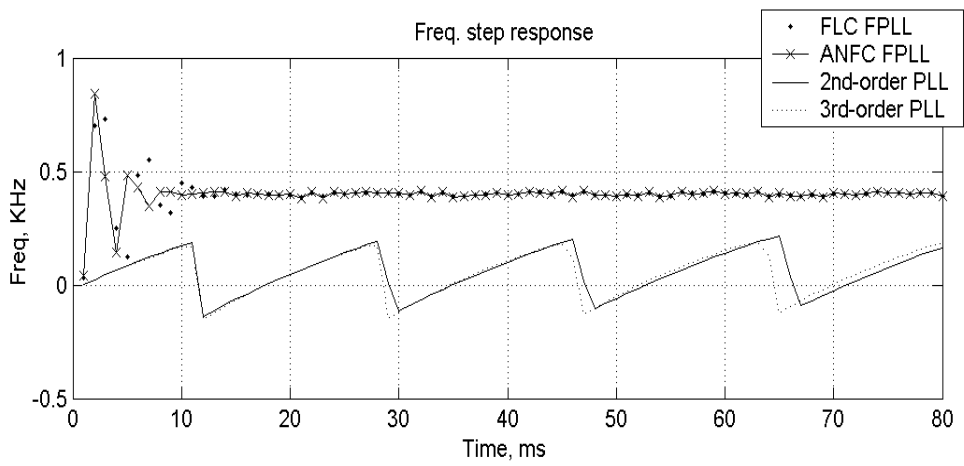

(b)

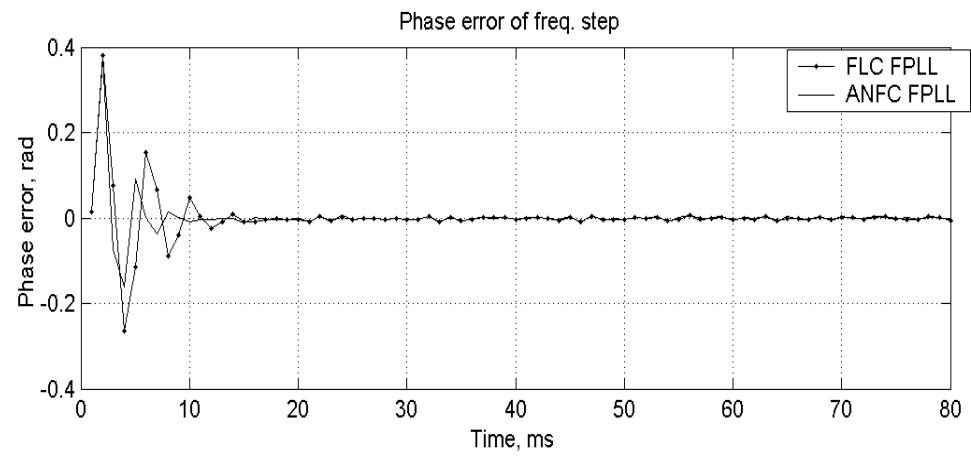

Figure 8. (a) Frequency step responses, (b) phase errors for each FLC FPLL, ANFC FPLL, secondorder PLL, and third-order PLL when input frequency offset is $400 \mathrm{~Hz}$. 
Table 4. Settling times for each digital carrier loop under Doppler frequency ramp environments

\begin{tabular}{|c|c|c|c|c|c|c|c|c|}
\hline $\begin{array}{l}\text { Gravitational } \\
\text { acceleration } \\
\text { (G) }\end{array}$ & 1 & 2 & 3 & 4 & 5 & 6 & 7 & 8 \\
\hline $\begin{array}{c}\text { Frequency } \\
\text { ramp } \\
\text { offset }(\mathrm{Hz} / \mathrm{s})\end{array}$ & 51.5 & 102.9 & 154.4 & 205.9 & 257.3 & 308.8 & 360.2 & 411.7 \\
\hline $\begin{array}{c}\text { Types of } \\
\text { digital } \\
\text { carrier loop }\end{array}$ & \multicolumn{8}{|c|}{ Settling time (ms) } \\
\hline FLC FPLL & 7 & 10 & 16 & 22 & 25 & 28 & 30 & 32 \\
\hline ANFC FPLL & 5 & 6 & 7 & 10 & 12 & 15 & 18 & 21 \\
\hline $\begin{array}{l}\text { Second-order } \\
\text { PLL }\end{array}$ & 128 & 198 & 250 & 287 & $\begin{array}{l}378 \\
(\mathrm{CS})\end{array}$ & LL & LL & LL \\
\hline $\begin{array}{c}\text { Third-order } \\
\text { PLL }\end{array}$ & 502 & 637 & 731 & $\begin{array}{l}822 \\
(\mathrm{CS})\end{array}$ & $\begin{array}{l}987 \\
(\mathrm{CS})\end{array}$ & LL & LL & LL \\
\hline
\end{tabular}

CS: cycle slips, LL: loss of lock.

the fuzzy controllers into the carrier loop, the locking range and pull-in range can be extended effectively.

We show in Figure 9 the simulation results of each method for a severe kinematic environment, i.e., frequency ramp tracking. The maximum rate of change of the Doppler frequency is set to be $400 \mathrm{~Hz} / \mathrm{s}$ as the dynamic input. In order to maintain lock on the carrier signal, both FI controller 1 and controller 2 are employed to track the phase waveform. The transient responses have a significant improvement in the convergence rate to the zero steady-state phase error as compared with the conventional ones. It is found that the settling times are 21 $\mathrm{ms}$ and $35 \mathrm{~ms}$ for ANFC and FLC FPLLs, respectively. Hence, the phase errors grow too rapidly, so that the conventional loops cannot pull in again and lose lock eventually. To demonstrate the benefit of our proposed loops, the motion acceleration of a vehicle ranging from $1 \mathrm{G}$ to $8 \mathrm{G}$ is simulated. Table 4 compares the simulated tracking performances of the fuzzy tracking loops with those of the conventional methods under these severe mobile cases. Clearly, traditional loops with constant noise bandwidth can only operate in smaller frequency ramp ranges (up to near $250 \mathrm{~Hz} / \mathrm{s}$ ). On the other hand, the fuzzy controller provides an adaptive gain in the DFLL and the acquisition performance of the dual-loop receiver can be significantly extended to $411.7 \mathrm{~Hz} / \mathrm{s}$. 
(a)

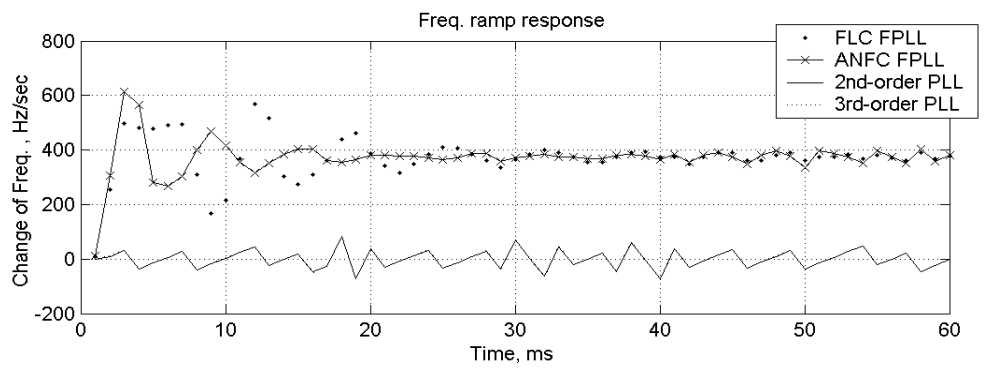

(b)

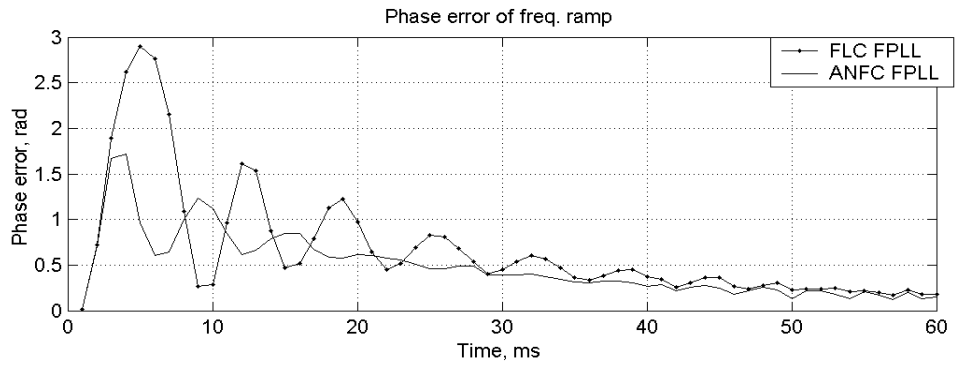

Figure 9. (a) Frequency ramp responses, (b) phase errors for each FLC FPLL, ANFC PLL, secondorder PLL, and third-order PLL when input frequency ramp is $400 \mathrm{~Hz} / \mathrm{s}$.

\section{Conclusions}

In this paper, a new method for improving the tracking performance of GPS receivers has been proposed. For dynamic surroundings, the FI scheme can control the dual-loop FPLL robustly and perform a better traceability of various phase signals during the transient period. For the FLC approach, the rule tables and membership functions are constructed on the basis of knowledge experience. In order to achieve an excellent tracking performance, the RLS algorithm is employed to optimize the ANF control method. Five kinds of carrier phase signals are considered for verification of dynamic trajectories. Numerical results over Monte Carlo simulations indicate that the FI carrier loops are capable of rapid acquisition speed and widening pull-in range compared to traditional loops. Both FLC and ANFC methods can achieve a short settling time while preventing the occurrence of cycle slips in kinematic environments. Moreover, the proposed FIbased method can be incorporated into a microcomputer system by software and can become a cost-effective implementation. 


\section{Acknowledgment}

The author thanks the National Science Council of the Republic of China, Taiwan, for financially supporting this research under contract no. NSC 94-2213-E-131004.

\section{References}

[1] R. E. Best, Phase-Locked Loops, Theory, Design and Applications, Mc-Graw-Hill, Inc., New York, 1993.

[2] M. S. Braasch and A. J. Van Dierendonck, GPS receiver architectures and measurements, Proceedings of IEEE, 1, pp. 48-64, January 1999.

[3] D. J. Jwo, Optimisation and sensitivity analysis of GPS receiver tracking loops in dynamic environments, IEE Proc. Rader, Sonar, Navig., 148, 4, August 2001.

[4] B. W. Parkinson and J. J. Spilker, Jr., Global Positioning System: Theory and Applications, AAAI, Washington, DC, 1996.

[5] D. Simon and H. El-Sherief, Fuzzy logic digital phase-locked loop filter design, IEEE Trans. Fuzzy Systems, 3, 2, 211-218, May 1995.

[6] J. B. Y. Tsui, Fundamentals of Global Positioning System Receivers, John Wiley \& Sons, Toronto, Canada, 2000. 